\title{
Gauge Invariant Treatment of the Energy Carried by a Gravitational Wave
}

\author{
Philip D. Mannheim \\ Department of Physics, University of Connecticut, Storrs, CT 06260*
}

(Dated: June 23, 2006)

\begin{abstract}
Even though the energy carried by a gravitational wave is not itself gauge invariant, the interaction with a gravitational antenna of the gravitational wave which carries that energy is. It therefore has to be possible to make some statements which involve the energy which are in fact gauge invariant, and it is the objective of this paper to provide them. In order to develop a gauge invariant treatment of the issues involved, we construct a specific action for gravitational fluctuations which is gauge invariant to second perturbative order. Then, via variation of this action, we obtain an energy-momentum tensor for perturbative gravitational fluctuations around a general curved background whose covariant conservation condition is also fully gauge invariant to second order. Contraction of this energy-momentum tensor with a Killing vector of the background conveniently allows us to convert this covariant conservation condition into an ordinary conservation condition which is also gauge invariant through second order. Then, via spatial integration we are able to obtain a relation involving the time derivative of the total energy of the fluctuation and its asymptotic spatial momentum flux which is also completely gauge invariant through second order. It is only in making the simplification of setting the asymptotic momentum flux to zero that one would actually lose manifest gauge invariance, with only invariance under those particular gauge transformations which leave the asymptotic momentum flux zero then remaining. However, if one works in an arbitrary gauge where the asymptotic momentum flux is non-zero, the gravitational wave will then deliver both energy and momentum to a gravitational antenna in a completely gauge invariant manner, no matter how badly behaved at infinity the gauge function might be.

PACS numbers: 95.30.Sf,04.20.-q,04.30.-w
\end{abstract}

*Electronic address: philip.mannheim@uconn.edu 


\section{INTRODUCTION}

In standard treatments of the energy carried by a gravitational fluctuation, the use of a non-covariant energy-momentum pseudo-tensor totally obscures the covariance and gauge issues involved, while additionally forcing one to only admit those particular gauge transformations which are are asymptotically flat. However, the full gauge invariance of general relativity equally holds for asymptotically badly-behaved gauge transformations as well, with the response of a gravitational antenna to a gravitational wave needing to be invariant under all gauge transformations both well- or badly-behaved if such a response is to be physically meaningful. Consequently, it is necessary to provide a treatment of gravitational fluctuations which takes the badly-behaved gauge transformations into account as well. Thus despite the fact that there is no generally covariant description of the energy carried by a gravitational wave (and not even one which is good to second perturbative order), one still has to be able to make gauge invariant statements regarding the interaction of the gravitational wave with a gravitational antenna and the energy which the gravitational wave transmits to it. It is thus the objective of this paper to provide a gauge invariant treatment of the issues involved, using an approach which retains full gauge invariance to second order at every step of the way. In particular, we develop a new technique (one based on a particularly chosen gaugeinvariant action for gravitational fluctuations) for constructing the energy-momentum tensor associated with a second order gravitational fluctuation. And even though the particular energy-momentum tensor we construct will not itself prove to be gauge invariant, because of the gauge invariance of our chosen fluctuation action, its covariant derivative nonetheless will be. However, the total energy and momentum carried by the gravitational wave are associated not with the fluctuation energy-momentum tensor itself but rather with the spatial integrals of its derivatives. Consequently, the gauge invariance of the covariant conservation condition for the energy-momentum tensor is all that we need in order to be able to obtain an integral relation which involves both the total energy and the total momentum of the gravitational wave which itself is fully gauge invariant through second order. The key point of this paper is thus that in order to secure the gauge invariance of the integral relations one does not actually need the gauge invariance of the fluctuation energy-momentum tensor itself but rather only that of its covariant conservation condition. Finally, once one has secured the gauge invariance of the integral relations, while one can then choose to work in a gauge 
in which the asymptotic momentum flux actually vanishes, the utility of our work is that we can instead go to some other (typically asymptotically badly-behaved) gauge in which the asymptotic momentum flux does not then vanish. In such a badly-behaved gauge the energy will then readjust since the relation between its time derivative and the asymptotic momentum flux is fully gauge invariant, with the gravitational wave then delivering both energy and momentum to a gravitational antenna in a fully gauge invariant manner. [1]

\section{FLUCTUATIONS IN FIRST ORDER}

If we start off knowing only that there is some general Einstein tensor $G^{\mu \nu}=R^{\mu \nu}-$ $(1 / 2) g^{\mu \nu} g^{\alpha \beta} R_{\alpha \beta}$ and some general energy-momentum tensor $T^{\mu \nu}$ both of which are independently covariantly conserved with respect to an arbitrary gravitational metric $g_{\mu \nu}$ (i.e. on non-stationary gravitational paths which are not required to obey the Einstein equations), the quantity $\Delta^{\mu \nu}=G^{\mu \nu}-\kappa_{4}^{2} T^{\mu \nu}$ will then be covariantly conserved even for gravitational paths which do not obey $\Delta^{\mu \nu}=0$. If we now break up all these various tensors into zeroth and first order parts so that the metric can be written as $g_{\mu \nu}=g_{\mu \nu}^{(0)}+g_{\mu \nu}^{(1)}=g_{\mu \nu}^{(0)}+h_{\mu \nu}$, $g^{\mu \nu}=g^{(0) \mu \nu}-h^{\mu \nu}$ (we use $h_{\mu \nu}$ to denote $\left.g_{\mu \nu}^{(1)}\right)$, the covariant conservation of the zeroth order $\Delta^{(0) \mu \nu}$ with respect to $g_{\mu \nu}^{(0)}$ (which we shall require) and the covariant conservation of the full $\Delta^{\mu \nu}$ with respect to the full $g_{\mu \nu}$ will then entail that the first order $\Delta^{(1) \mu \nu}$ as defined as

$$
\Delta_{\mu \nu}^{(1)}=R_{\mu \nu}^{(1)}-\frac{1}{2} h_{\mu \nu} g^{(0) \alpha \beta} R_{\alpha \beta}^{(0)}-\frac{1}{2} g_{\mu \nu}^{(0)} g^{(0) \alpha \beta} R_{\alpha \beta}^{(1)}+\frac{1}{2} g_{\mu \nu}^{(0)} h^{\alpha \beta} R_{\alpha \beta}^{(0)}-\kappa_{4}^{2} T_{\mu \nu}^{(1)}
$$

will then obey

$$
\partial_{\nu} \Delta^{(1) \mu \nu}+\Delta^{(1) \mu \lambda} \Gamma_{\nu \lambda}^{(0) \nu}+\Delta^{(1) \nu \lambda} \Gamma_{\nu \lambda}^{(0) \mu}+\Delta^{(0) \mu \lambda} \Gamma_{\nu \lambda}^{(1) \nu}+\Delta^{(0) \nu \lambda} \Gamma_{\nu \lambda}^{(1) \mu}=0
$$

where $\Gamma_{\nu \lambda}^{(1) \mu}$ and $R_{\mu \nu}^{(1)}$ are given by $\Gamma_{\nu \lambda}^{(1) \mu}=(1 / 2) g^{(0) \mu \rho}\left(\nabla_{\lambda} h_{\rho \nu}+\nabla_{\nu} h_{\rho \lambda}-\nabla_{\rho} h_{\nu \lambda}\right)$ and $R_{\mu \nu}^{(1)}=(1 / 2)\left(\nabla_{\mu} \nabla_{\nu} h-\nabla_{\alpha} \nabla_{\mu} h_{\nu}^{\alpha}-\nabla_{\alpha} \nabla_{\nu} h_{\mu}^{\alpha}+\nabla_{\alpha} \nabla^{\alpha} h_{\mu \nu}\right)$, and where the covariant $\nabla_{\mu}$

derivatives are evaluated with respect to the zeroth order metric $g_{\mu \nu}^{(0)}$. For stationary zeroth order paths which obey a zeroth order Einstein equation $\Delta_{\mu \nu}^{(0)}=0$, it then follows that all first order paths will obey

$$
\nabla_{\nu} \Delta^{(1) \mu \nu}=0
$$

even without the imposition of any equation of motion for the first order $h_{\mu \nu}$. As introduced, the quantity $\Delta^{(1) \mu \nu}$ transforms as a true tensor with respect to the zeroth order $g_{\mu \nu}^{(0)}$, and 
remains unchanged if $h_{\mu \nu}$ is replaced by $\bar{h}_{\mu \nu}=h_{\mu \nu}+\nabla_{\mu} \epsilon_{\nu}+\nabla_{\nu} \epsilon_{\mu}$, with the $\nabla_{\nu} \Delta^{(1) \mu \nu}=0$ condition thus being gauge invariant to first order in $\epsilon_{\mu}$.

The value of breaking $\Delta^{\mu \nu}$ into zeroth and first order paths, is that if we perturb the zeroth order background $g_{\mu \nu}^{(0)}$ with some first order perturbation $\tau_{\mu \nu}^{(1)}$ which is also conserved with respect to the background, the perturbation will induce changes in both the background Einstein tensor and the background energy-momentum tensor, with the first order $h_{\mu \nu}$ then being fixed as the solution to

$$
\Delta_{\mu \nu}^{(1)}=-\kappa_{4}^{2} \tau_{\mu \nu}^{(1)}
$$

once the first order Einstein equations are imposed. As such, Eq. (4) is automatically fully gauge invariant to first order in $\epsilon_{\mu}$.

\section{FLUCTUATIONS IN SECOND ORDER}

The presence of the perturbation will also lead to a second order effect, namely the emission of a gravitational wave, and Weinberg 2] has suggested that we identify its energymomentum tensor as $\left(1 / \kappa_{4}^{2}\right.$ times $)$ that part, viz. $\Delta_{\mu \nu}^{(2)}(h)$, of the full $\Delta_{\mu \nu}$ which is second order in $h_{\mu \nu}$. Since the Einstein equations take the form

$$
\Delta_{\mu \nu}^{(0)}+\Delta_{\mu \nu}^{(1)}+\Delta_{\mu \nu}^{(2)}=-\kappa_{4}^{2} \tau_{\mu \nu}^{(1)}
$$

through second order, in solutions to Eq. (15) which obey both $\Delta_{\mu \nu}^{(0)}=0$ and $\Delta_{\mu \nu}^{(1)}=-\kappa_{4}^{2} \tau_{\mu \nu}^{(1)}$, the second order $\Delta_{\mu \nu}^{(2)}$ and $\nabla_{\nu} \Delta^{(2) \mu \nu}$ (as evaluated with respect to the background $g_{\mu \nu}^{(0)}$ ) would both have to vanish identically. Since the full second order $\Delta_{\mu \nu}^{(2)}$ does vanish, the only way for that the piece of it which is second order in $h_{\mu \nu}$ to not itself vanish when $h_{\mu \nu}$ is itself a solution to the first order Eq. (44) is if in addition to $\Delta_{\mu \nu}^{(2)}(h)$, the full $\Delta_{\mu \nu}^{(2)}$ contains some other, intrinsically second order, term [to be labelled $\Delta_{\mu \nu}^{(2)}\left(g^{(2)}\right)$ ] which would have to be equal to $-\Delta_{\mu \nu}^{(2)}(h)$. However, in that case it would only be the conservation of the sum of $\Delta_{\mu \nu}^{(2)}(h)$ and $\Delta_{\mu \nu}^{(2)}\left(g^{(2)}\right)$ which would be secured by the imposition of Eq. (15), to thus not immediately ensure that $\Delta_{\mu \nu}^{(2)}(h)$ itself would in fact be able to serve as a conserved gravitational wave energy-momentum tensor. However, as we now show, on explicitly constructing the additional $\Delta_{\mu \nu}^{(2)}\left(g^{(2)}\right)$ term in the explicit case of fluctuations around a flat background, we will find it to be conserved all on its own, so that it does not in fact exchange energy and momentum with $\Delta_{\mu \nu}^{(2)}(h)$, to thereby allow $\Delta_{\mu \nu}^{(2)}(h)$ to be independently conserved after all. 
Then, guided by this decoupling of the $h_{\mu \nu}$ and $g_{\mu \nu}^{(2)}$ sectors in the flat background case, using an action principle we shall then generalize the decoupling to general curved backgrounds as well. [3]

When we perturb a system with a first order perturbation $\tau_{\mu \nu}^{(1)}$ we not only induce a first order change in the metric, we will also induce higher order changes in it as well. To second order then we must take the perturbed metric to be of the form

$$
g_{\mu \nu}=g_{\mu \nu}^{(0)}+h_{\mu \nu}+g_{\mu \nu}^{(2)}
$$

Through second order the associated inverse metric and determinant are given by

$$
g^{\mu \nu}=g^{(0) \mu \nu}-h^{\mu \nu}+h^{\mu}{ }_{\sigma} h^{\sigma \nu}-g^{(2) \mu \nu} \quad, \quad g=g^{(0)}\left(1+h+\frac{h^{2}}{2}-\frac{h_{\mu \nu} h^{\mu \nu}}{2}+g^{(0) \mu \nu} g_{\mu \nu}^{(2)}\right)
$$

with the second order term in the Einstein tensor being given by

$$
\begin{aligned}
G_{\mu \nu}^{(2)}= & R_{\mu \nu}^{(2)}-\frac{1}{2} g_{\mu \nu}^{(0)}\left(g^{(0) \alpha \beta} R_{\alpha \beta}^{(2)}-h^{\alpha \beta} R_{\alpha \beta}^{(1)}+h^{\alpha}{ }_{\sigma} h^{\sigma \beta} R_{\alpha \beta}^{(0)}-g^{(2) \alpha \beta} R_{\alpha \beta}^{(0)}\right) \\
& -\frac{1}{2} h_{\mu \nu}\left(g^{(0) \alpha \beta} R_{\alpha \beta}^{(1)}-h^{\alpha \beta} R_{\alpha \beta}^{(0)}\right)-\frac{1}{2} g_{\mu \nu}^{(2)} g^{(0) \alpha \beta} R_{\alpha \beta}^{(0)} .
\end{aligned}
$$

To identify the specific role played by the intrinsically second order $g_{\mu \nu}^{(2)}$ it is sufficient to descend to the flat background case where $g_{\mu \nu}^{(0)}=\eta_{\mu \nu}$. On recalling that the general curved space Riemann tensor is given by $R_{\lambda \mu \nu \kappa}=(1 / 2)\left(\partial_{\kappa} \partial_{\mu} g_{\lambda \nu}-\partial_{\kappa} \partial_{\lambda} g_{\mu \nu}-\partial_{\nu} \partial_{\mu} g_{\lambda \kappa}+\partial_{\nu} \partial_{\lambda} g_{\mu \kappa}\right)+$ $g_{\eta \sigma}\left(\Gamma_{\nu \lambda}^{\eta} \Gamma_{\mu \kappa}^{\sigma}-\Gamma_{\kappa \lambda}^{\eta} \Gamma_{\mu \nu}^{\sigma}\right)$, in the flat background case we see that the $g_{\mu \nu}^{(2)}$ dependent term in Eq. (8) is given by

$$
\begin{aligned}
G_{\mu \nu}^{(2)}\left(g^{(2)}\right)= & \frac{1}{2}\left(\partial_{\mu} \partial_{\nu} g_{\alpha}^{(2) \alpha}-\partial_{\alpha} \partial_{\mu} g_{\nu}^{(2) \alpha}-\partial_{\alpha} \partial_{\nu} g_{\mu}^{(2) \alpha}+\partial_{\alpha} \partial^{\alpha} g_{\mu \nu}^{(2)}\right) \\
& -\frac{1}{2} \eta_{\mu \nu}\left(\partial_{\alpha} \partial^{\alpha} g_{\beta}^{(2) \beta}-\partial_{\alpha} \partial_{\beta} g^{(2) \alpha \beta}\right) .
\end{aligned}
$$

As such this expression is quite remarkable. Specifically it says that the dependence of the second order $G_{\mu \nu}^{(2)}\left(g^{(2)}\right)$ on $g_{\mu \nu}^{(2)}$ is precisely the same as the dependence of the first order $G_{\mu \nu}^{(1)}$ on $h_{\mu \nu}\left[\operatorname{viz} . G_{\mu \nu}^{(1)}=(1 / 2)\left(\partial_{\mu} \partial_{\nu} h-\partial_{\alpha} \partial_{\mu} h^{\alpha}{ }_{\nu}-\partial_{\alpha} \partial_{\nu} h_{\mu}^{\alpha}+\partial_{\alpha} \partial^{\alpha} h_{\mu \nu}\right)-(1 / 2) \eta_{\mu \nu}\left(\partial_{\alpha} \partial^{\alpha} h-\partial_{\alpha} \partial_{\beta} h^{\alpha \beta}\right)\right]$ in the same flat background. However, since $G_{\mu \nu}^{(1)}$ kinematically obeys a linearized Bianchi identity without any need to impose any equation of motion, it follows that $G_{\mu \nu}^{(2)}\left(g^{(2)}\right)$ must do so too, and thus we conclude that the condition $\partial_{\nu} G^{(2) \mu \nu}\left(g^{(2)}\right)=0$ not only holds, but that it does so without needing to impose any stationarity condition on $g_{\mu \nu}^{(2)}$ whatsoever. If however, we now do impose Eq. (15), we will then find that $G_{\mu \nu}^{(2)}(h)$ (and thus $\Delta_{\mu \nu}^{(2)}(h)$ in the 
flat background case) will be conserved also. After the fact then we conclude that in the flat background case we can set

$$
\partial_{\nu} \Delta^{(2) \mu \nu}(h)=0
$$

after all, just as we want.

An additional feature of the form of Eq. (9) is that once we have fixed $h_{\mu \nu}$ from the first order Eq. (41), the vanishing of the full $\Delta_{\mu \nu}^{(2)}$ in solutions to Eq. (15) would then enable us to determine $g_{\mu \nu}^{(2)}$ as a closed form function which would indeed be quadratic in $h_{\mu \nu}$, just as it should be. And not only that, from the explicit form of Eq. (9), we see that the equation for $g_{\mu \nu}^{(2)}$ would be in the form of none other than an Einstein equation whose source term is $\Delta_{\mu \nu}^{(2)}(h)$. Finally, with the change in the second order $g_{\mu \nu}^{(2)}$ under an infinitesimal gauge transformation $x^{\mu} \rightarrow x^{\mu}-\epsilon^{\mu}$ being given by $g_{\mu \nu}^{(2)} \rightarrow g_{\mu \nu}^{(2)}+h_{\mu \lambda}(x) \partial_{\nu} \epsilon^{\lambda}+h_{\nu \lambda}(x) \partial_{\mu} \epsilon^{\lambda}+$ $\epsilon^{\lambda} \partial_{\lambda} h_{\mu \nu}(x)+\partial_{\mu}\left(\epsilon^{\lambda} \partial_{\lambda} \epsilon_{\nu}\right)+\partial_{\nu}\left(\epsilon^{\lambda} \partial_{\lambda} \epsilon_{\mu}\right)+\partial_{\mu} \epsilon_{\lambda} \partial_{\nu} \epsilon^{\lambda}$, we infer that the vanishing of $\partial_{\nu} \Delta^{(2) \mu \nu}\left(g^{(2)}\right)$ is itself gauge invariant, with the vanishing of $\partial_{\nu} \Delta^{(2) \mu \nu}(h)$ then being gauge invariant through second order too. Thus even though $\Delta^{(2) \mu \nu}(h)$ is not itself gauge invariant, its derivative is, with the associated integral condition

$$
\frac{\partial}{\partial t} \int d^{3} x \Delta^{(2) 00}(h)=-\int d S n_{i} \Delta^{(2) 0 i}(h)
$$

then being gauge invariant too 4]. Without any loss of gauge invariance one can thus arrive at an integral relation which relates the time derivative of the energy to an asymptotic momentum flux, no matter how badly behaved a gauge one might choose to work in. The only place where gauge invariance could be lost would be in dropping the asymptotic momentum flux term, as its vanishing does not occur in asymptotically badly-behaved gauges. Nonetheless, in gauges where the asymptotic momentum flux is non-vanishing, the gravitational wave would deliver not just energy but momentum also to a gravitational antenna, doing so in a completely gauge invariant manner [5].

\section{THE REASON FOR THE DECOUPLING}

To understand and to then be able to generalize the above found decoupling of the two second order sectors, we recall a very useful property of the Einstein-Hilbert action $I_{\mathrm{EH}}=-\left(1 / 2 \kappa_{4}^{2}\right) \int d^{4} x(-g)^{1 / 2} R_{\alpha}^{\alpha}$, namely that under integration by parts it can brought to 
the form [6]

$$
I_{\mathrm{EH}}=\frac{1}{2 \kappa_{4}^{2}} \int(-g)^{1 / 2} g^{\mu \nu}\left(\Gamma_{\mu \beta}^{\alpha} \Gamma_{\nu \alpha}^{\beta}-\Gamma_{\mu \nu}^{\alpha} \Gamma_{\alpha \beta}^{\beta}\right)
$$

For our purposes here the great utility of Eq. (12) is that for a flat background an expansion of $I_{\mathrm{EH}}$ through second order can only involve terms which are no higher than first order in the Christoffel symbols, to thus involve $h_{\mu \nu}$ but not $g_{\mu \nu}^{(2)}$ at all. The entire dependence of $R_{\alpha \beta}^{(2)}$ on $g_{\mu \nu}^{(2)}$ can thus be removed from the second order $I_{\mathrm{EH}}^{(2)}$ by an integration by parts which would then put the $g_{\mu \nu}^{(2)}$ dependence entirely in irrelevant surface terms. Further, on explicitly evaluating Eq. (12) in a flat background, $I_{\mathrm{EH}}^{(2)}$ is found to take the form

$$
\begin{aligned}
I_{\mathrm{EH}}^{(2)}= & \frac{1}{8 \kappa_{4}^{2}} \int d^{4} x h^{\mu \nu}\left(\partial_{\mu} \partial_{\nu} h-\partial_{\alpha} \partial_{\mu} h_{\nu}^{\alpha}-\partial_{\alpha} \partial_{\nu} h_{\mu}^{\alpha}\right. \\
& \left.+\partial_{\alpha} \partial^{\alpha} h_{\mu \nu}-\eta_{\mu \nu} \partial_{\alpha} \partial^{\alpha} h+\eta_{\mu \nu} \partial_{\alpha} \partial_{\beta} h^{\alpha \beta}\right) .
\end{aligned}
$$

We recognize Eq. (13) to be of none other than the form

$$
I_{\mathrm{EH}}^{(2)}=\frac{1}{4 \kappa_{4}^{2}} \int d^{4} x h^{\mu \nu} G_{\mu \nu}^{(1)}
$$

where $G_{\mu \nu}^{(1)}=R_{\mu \nu}^{(1)}-(1 / 2) \eta_{\mu \nu} R^{(1)}$ is the first order change in the Einstein tensor in the flat background. As such, the stationary variation of Eq. (14) with respect to the fluctuation $h_{\mu \nu}$ would thus yield the source free region version of Eq. (44) as evaluated in a flat background, viz. the first order $G_{\mu \nu}^{(1)}=0$. As an action, the second order $I_{\mathrm{EH}}^{(2)}$ is gauge invariant under $h_{\mu \nu} \rightarrow h_{\mu \nu}+\partial_{\mu} \epsilon_{\nu}+\partial_{\nu} \epsilon_{\mu}$ since $G_{\mu \nu}^{(1)}$ is itself gauge invariant and $\partial_{\mu} G^{(1) \mu \nu}$ is kinematically zero, and thus has to lead to a first order wave equation $G_{\mu \nu}^{(1)}=0$ which is gauge invariant too. In other words, since the first order wave equation is gauge invariant, there has to exist some second order action from which it can be derived, an action which would itself need to be gauge invariant to second order in $\epsilon_{\mu}$. Moreover, since the first order wave equation cannot depend on the second order $g_{\mu \nu}^{(2)}$, the requisite gauge invariant second order action could not depend on $g_{\mu \nu}^{(2)}$ either. Via Eq. (14) then discussion of the sector which is second order in $h_{\mu \nu}$ can thus be conducted without reference to $g_{\mu \nu}^{(2)}$ at all.

Since the $I_{\mathrm{EH}}^{(2)}$ action provides us with an equation of motion when we vary with respect to the fluctuation $h_{\mu \nu}$, we can view $I_{\mathrm{EH}}^{(2)}$ as describing a field theory in which a spin two field $h_{\mu \nu}$ propagates in some background $\eta_{\mu \nu}$. For such a spin two field theory we can construct an energy-momentum tensor. Specifically, in Eq. (113) we replace the metric $\eta_{\mu \nu}$ by a general $g_{\mu \nu}$, replace ordinary derivatives by covariant ones, and then do a functional variation of the 
$I_{\mathrm{EH}}^{(2)}$ action with respect to $g_{\mu \nu}$ to construct the rank two tensor $t^{(2) \mu \nu}=\left(2 /(-g)^{1 / 2}\right) \delta I_{\mathrm{EH}}^{(2)} / \delta g_{\mu \nu}$, with the flat space limit of this $t_{\mu \nu}^{(2)}$ then being the requisite flat spacetime energy-momentum tensor associated with the propagation of $h_{\mu \nu}$ in the flat background. Moreover, with the action $I_{\mathrm{EH}}^{(2)}$ being a general coordinate scalar, in solutions to the first order $h_{\mu \nu}$ wave equation the second order $t_{\mu \nu}^{(2)}$ constructed this way will automatically be covariantly conserved.

To actually perform the requisite variation of the action of Eqs. (13) and (14), we must vary with respect to $g_{\mu \nu}$ without yet imposing the first order flat background $G_{\mu \nu}^{(1)}=0$ equation of motion for $h_{\mu \nu}$, with a fair amount of algebra then being found to yield an associated $t_{\mu \nu}^{(2)}$ of the form

$$
\begin{aligned}
4 \kappa_{4}^{2} t_{\mu \nu}^{(2)}= & h^{\alpha \beta} \partial_{\alpha} \partial_{\mu} h_{\nu \beta}+h^{\alpha \beta} \partial_{\alpha} \partial_{\nu} h_{\mu \beta}+h_{\mu \alpha} \partial_{\nu} \partial_{\beta} h^{\alpha \beta}+h_{\nu \alpha} \partial_{\mu} \partial_{\beta} h^{\alpha \beta}-2 \eta_{\mu \nu} h^{\alpha \beta} \partial_{\alpha} \partial_{\sigma} h_{\beta}^{\sigma} \\
& -h_{\mu \alpha} \partial_{\beta} \partial^{\beta} h^{\alpha}{ }_{\nu}-h_{\nu \alpha} \partial_{\beta} \partial^{\beta} h^{\alpha}{ }_{\mu}-h_{\mu \alpha} \partial_{\nu} \partial^{\alpha} h-h_{\nu \alpha} \partial_{\mu} \partial^{\alpha} h+h_{\mu \nu} \partial_{\alpha} \partial^{\alpha} h \\
& +\eta_{\mu \nu} h^{\alpha \beta} \partial_{\alpha} \partial_{\beta} h+\partial_{\mu} h_{\nu \alpha} \partial_{\beta} h^{\alpha \beta}+\partial_{\nu} h_{\mu \alpha} \partial_{\beta} h^{\alpha \beta}-\partial_{\mu} h^{\alpha \beta} \partial_{\alpha} h_{\nu \beta}-\partial_{\nu} h^{\alpha \beta} \partial_{\alpha} h_{\mu \beta} \\
& -2 \partial_{\alpha} h^{\alpha}{ }_{\mu} \partial_{\beta} h^{\beta}{ }_{\nu}+2 \partial_{\alpha} h_{\mu \nu} \partial_{\beta} h^{\alpha \beta}-\eta_{\mu \nu} \partial_{\alpha} h^{\alpha}{ }_{\beta} \partial_{\sigma} h^{\beta \sigma}+\partial_{\mu} h^{\alpha \beta} \partial_{\nu} h_{\alpha \beta}-\frac{1}{2} \eta_{\mu \nu} \partial_{\sigma} h_{\alpha \beta} \partial^{\sigma} h^{\alpha \beta} \\
& +\partial_{\alpha} h^{\alpha}{ }_{\mu} \partial_{\nu} h+\partial_{\alpha} h^{\alpha}{ }_{\nu} \partial_{\mu} h-\partial_{\alpha} h_{\mu \nu} \partial^{\alpha} h-\partial_{\mu} h \partial_{\nu} h+\frac{1}{2} \eta_{\mu \nu} \partial_{\alpha} h \partial^{\alpha} h .
\end{aligned}
$$

With the covariant derivative of this $t^{(2) \mu \nu}$ evaluating to

$$
\begin{aligned}
4 \kappa_{4}^{2} \partial_{\mu} t^{(2) \mu \nu}= & \left(\partial^{\nu} h^{\mu \alpha}-2 \partial^{\mu} h^{\nu \alpha}\right)\left[\partial_{\beta} \partial^{\beta} h_{\mu \alpha}-\partial_{\mu} \partial_{\beta} h_{\alpha}^{\beta}-\partial_{\alpha} \partial_{\beta} h_{\mu}^{\beta}+\partial_{\mu} \partial_{\alpha} h\right. \\
& \left.-\eta_{\mu \alpha}\left(\partial_{\beta} \partial^{\beta} h-\partial_{\beta} \partial_{\sigma} h^{\beta \sigma}\right)\right]=2\left(\partial^{\nu} h^{\mu \alpha}-2 \partial^{\mu} h^{\nu \alpha}\right) G_{\mu \alpha}^{(1)}
\end{aligned}
$$

we readily confirm that for any on-shell $h_{\mu \nu}$ which then does obey the first order equation of motion $G_{\mu \nu}^{(1)}=0$, the second order energy-momentum tensor does indeed obey $\partial_{\mu} t^{(2) \mu \nu}=0$, just as it should. Under a gauge transformation of the form $h_{\mu \nu} \rightarrow h_{\mu \nu}+\partial_{\mu} \epsilon_{\nu}+\partial_{\nu} \epsilon_{\mu}$, we note that while $t^{(2) \mu \nu}$ itself will then acquire terms which are both linear and quadratic in $\epsilon_{\mu}$, because of the gauge invariance of $G_{\mu \nu}^{(1)}$, the covariant derivative of $t^{(2) \mu \nu}$ will only acquire a term which is linear in $\epsilon_{\mu}$, viz. the term $-4\left[\partial^{\mu} \partial^{\alpha} \epsilon^{\nu}\right] G_{\mu \alpha}^{(1)}$. With this specific term vanishing when $G_{\mu \nu}^{(1)}$ vanishes, and with $G_{\mu \nu}^{(1)}$ vanishing for every $h_{\mu \nu}+\partial_{\mu} \epsilon_{\nu}+\partial_{\nu} \epsilon_{\mu}$ if it already vanishes for any given $h_{\mu \nu}$, the on-shell vanishing of $\partial_{\mu} t^{(2) \mu \nu}$ is thus seen to be fully gauge invariant to second order in $\epsilon_{\mu}$.

Some simplification of Eq. (15) can be obtained by working in the convenient harmonic gauge where $\partial_{\nu} h^{\mu \nu}-(1 / 2) \partial^{\mu} h=0$, with $t^{(2) \mu \nu}$ then reducing to []

$$
4 \kappa_{4}^{2} t_{\mu \nu}^{(2)}=-\partial_{\mu} h^{\alpha \beta} \partial_{\alpha} h_{\nu \beta}-\partial_{\nu} h^{\alpha \beta} \partial_{\alpha} h_{\mu \beta}+\partial_{\mu} h^{\alpha \beta} \partial_{\nu} h_{\alpha \beta}+\frac{1}{4} \eta_{\mu \nu} \partial_{\alpha} h \partial^{\alpha} h
$$




$$
\begin{aligned}
& +h^{\alpha \beta} \partial_{\alpha} \partial_{\mu} h_{\nu \beta}+h^{\alpha \beta} \partial_{\alpha} \partial_{\nu} h_{\mu \beta}-\frac{1}{2} h_{\mu \alpha} \partial_{\nu} \partial^{\alpha} h-\frac{1}{2} h_{\nu \alpha} \partial_{\mu} \partial^{\alpha} h \\
& -\frac{1}{2} \eta_{\mu \nu} \partial_{\sigma} h_{\alpha \beta} \partial^{\sigma} h^{\alpha \beta}+\frac{1}{2} \partial_{\mu} h_{\nu \alpha} \partial^{\alpha} h+\frac{1}{2} \partial_{\nu} h_{\mu \alpha} \partial^{\alpha} h-\frac{1}{2} \partial_{\mu} h \partial_{\nu} h \\
& -h_{\mu \alpha} \partial_{\beta} \partial^{\beta} h_{\nu}^{\alpha}-h_{\nu \alpha} \partial_{\beta} \partial^{\beta} h_{\mu}^{\alpha}+h_{\mu \nu} \partial_{\alpha} \partial^{\alpha} h .
\end{aligned}
$$

With a typical box-normalized solution to the harmonic gauge wave equation $\partial_{\alpha} \partial^{\alpha} h_{\mu \nu}=$ $(1 / 2) \eta_{\mu \nu} \partial_{\alpha} \partial^{\alpha} h$ being of the form $h_{\mu \nu}=2 \kappa_{4} e^{i p \cdot x} e_{\mu \nu}\left(p^{\lambda}\right) /\left(2 p^{0}\right)^{1 / 2} L^{3 / 2}+$ c.c. where $p_{\mu} p^{\mu}=0$ and where the polarization tensor obeys $p_{\nu} e^{\mu \nu}=(1 / 2) p^{\mu} e^{\alpha}{ }_{\alpha}$, in such solutions the asymptotic momentum flux is found to vanish, with the on-shell fluctuation energy then being found to be given by the time-independent

$$
E^{(2)}=\int d^{3} x t^{(2) 00}=p^{0}\left[e^{\alpha \beta} e_{\alpha \beta}-\frac{1}{2}\left(e_{\alpha}^{\alpha}\right)^{2}\right],
$$

just as one would want of an energy [8]. Interestingly, the value obtained for $E^{(2)}$ is precisely the same as that which would be obtained via the relevant Weinberg prescription, viz. $\left(1 / \kappa_{4}^{2}\right) \int d^{3} x\left[R^{(2) 00}-(1 / 2) \eta^{00} R_{\alpha}^{(2) \alpha}\right]$, when evaluated under exactly the same conditions $[9]$.

\section{GENERAL ENERGY-MOMENTUM TENSOR}

In order to extend the above analysis to a general curved space background, we first need to find a fluctuation energy-momentum tensor which is covariantly conserved, and then need to manipulate the covariant conservation condition in a way which will yield an ordinary conservation condition while not losing gauge invariance. As regards the first issue, the solution is immediately at hand, since variation with respect to $h_{\mu \nu}$ of the fully covariant extension of Eq. (14), viz.

$$
I_{\mathrm{EH}}^{(2)}=\frac{1}{4 \kappa_{4}^{2}} \int d^{4} x(-g)^{1 / 2} h^{\mu \nu} \Delta_{\mu \nu}^{(1)}
$$

leads directly to $\Delta_{\mu \nu}^{(1)}=0$. With the energy-momentum tensor $t^{(2) \mu \nu}=\left(2 /(-g)^{1 / 2}\right) \delta I_{\mathrm{EH}}^{(2)} / \delta g_{\mu \nu}$ constructed from this $I_{\mathrm{EH}}^{(2)}$ being covariantly conserved when $\Delta_{\mu \nu}^{(1)}=0$, and with $I_{\mathrm{EH}}^{(2)}$ being invariant under $h_{\mu \nu} \rightarrow h_{\mu \nu}+\nabla_{\mu} \epsilon_{\nu}+\nabla_{\nu} \epsilon_{\mu}$, the $\nabla_{\nu} t^{(2) \mu \nu}=0$ condition is thus fully gauge invariant to second order.

To use the covariant conservation condition to extract an ordinary one we follow Abbot and Deser [10] and contract the general $t^{(2) \mu \nu}$ with a Killing vector $K_{\nu}$ of the curved background [1]]. With Killing vectors obeying the antisymmetric $\nabla_{\mu} K_{\nu}=-\nabla_{\nu} K_{\mu}$, and 
with $t^{(2) \mu \nu}$ being symmetric (our very construction of it as $\left(2 /(-g)^{1 / 2}\right) \delta I_{\mathrm{EH}}^{(2)} / \delta g_{\mu \nu}$ obliges it to be symmetric), the covariant conservation of the 4 -vector $J^{\mu}=t^{(2) \mu \nu} K_{\nu}$ immediately follows since $\nabla_{\mu} J^{\mu}=\left[\nabla_{\mu} t^{(2) \mu \nu}\right] K_{\nu}+t^{(2) \mu \nu} \nabla_{\mu} K_{\nu}=0$. Consequently, with $\nabla_{\mu} J^{\mu}=$ $(-g)^{-1 / 2} \partial_{\mu}\left[(-g)^{1 / 2} J^{\mu}\right]$, Eq. (11) generalizes to

$$
\begin{aligned}
\frac{\partial}{\partial t} \int d^{3} x(-g)^{1 / 2} t^{(2) 0 \nu} K_{\nu} & =-\int d^{3} x \frac{\partial\left[(-g)^{1 / 2} t^{(2) i \nu} K_{\nu}\right]}{\partial x^{i}} \\
& =-\int d S n_{i}(-g)^{1 / 2} t^{(2) i \nu} K_{\nu}
\end{aligned}
$$

to yield the gauge invariant integral relation we seek [12].

To illustrate the utility of our formalism, we apply it to the recently introduced $A d S_{5} / Z_{2}$ based brane world of Randall and Sundrum [13]. In a brane world with maximally 4symmetric branes the background geometry is taken to be of the separable form $d s^{2}=$ $d w^{2}+e^{2 A(|w|)} q_{\mu \nu} d x^{\mu} d x^{\nu}$ where $w$ is the fifth coordinate, $A(|w|)$ is the so-called warp factor, and the $w$-independent $q_{\mu \nu}$ is the induced metric on the brane. One is interested in the propagation in this background of axial gauge, transverse-traceless tensor fluctuations which obey the first order wave equation $\Delta G_{M N}^{(1)}=(1 / 2)\left[\nabla_{A} \nabla^{A} h_{M N}+2 b^{2} h_{M N}\right]=0$ where $-b^{2}$ is the curvature of $A d S_{5}$ and $M=(0,1,2,3,5)$. Calculation of the $t^{(2) M N}$ associated with the 5-dimensional action $I_{\mathrm{EH}}^{(2)}=\left(1 / 4 \kappa_{5}^{2}\right) \int d^{5} x(-g)^{1 / 2} h^{M N} \Delta G_{M N}^{(1)}$ is rather lengthy, with it being found [14] to take the form

$$
\begin{aligned}
4 \kappa_{5}^{2} t^{(2) M N}= & h_{A}^{B} \nabla_{B} \nabla^{M} h^{N A}+h_{A}^{B} \nabla_{B} \nabla^{N} h^{M A}+\nabla^{M} h^{A B} \nabla^{N} h_{A B}-\frac{1}{2} g^{M N} \nabla^{S} h^{A B} \nabla_{S} h_{A B} \\
& -\nabla^{M} h^{A B} \nabla_{B} h_{A}^{N}-\nabla^{N} h^{A B} \nabla_{B} h_{A}^{M}+b^{2} g^{M N} h^{A B} h_{A B}+10 b^{2} h^{M A} h_{A}^{N}
\end{aligned}
$$

on shell, with $\nabla_{M} t^{(2) M N}$ indeed being found to vanish identically in modes which obey $\nabla_{A} \nabla^{A} h_{M N}+2 b^{2} h_{M N}=0$. Separable mode solutions to the wave equation have a dependence on $|w|$ of the generic form $f_{m}(|w|)$ where $m$ is a separation constant, so that with $K_{M}=(-1,0,0,0,0)$ being a timelike $A d S_{5}$ Killing vector, modes with a vanishing asymptotic momentum flux $t^{(2) 05}$ will then have a time-independent energy. For the case where the induced metric on the brane is an $M_{4}$ or $A d S_{4}$ geometry, $t^{(2) 05}$ is found to behave asymptotically as $f_{m}(|w|)\left[f_{m}^{\prime}(|w|)-2 A^{\prime} f_{m}(|w|)\right]$, with the vanishing of this quantity leading to a time-independent energy whose dependence on the fifth coordinate is given by $\int_{0}^{\infty} d|w| e^{-2 A} f_{m}^{2}(|w|)[15]$. With the energy being a bilinear function of $h_{\mu \nu}$, we recognize the finiteness of this integral as being none other than the normalization condition which is 
ordinarily used in the brane world 16], just as needed to enable us to construct a propagator with which to integrate Eq. (41) [17], [18].

\section{Acknowledgments}

This work grew out of a study of brane-world fluctuations in which the author was engaged with Dr. A. H. Guth, Dr. D. I. Kaiser and Dr. A. Nayeri, and the author would like to thank them for their many helpful comments.

[1] While there is a quite extensive literature on studies of gravitational fluctuations through second order [see e.g. M. Bruni, S. Matarrese, S. Mollerach and S. Sonego, Class. Quant. Grav. 14, 2585 (1997); K. Nakamura, Prog. Theor. Phys. 110, 723 (2003); K. A. Malik and D. Wands, Class. Quant. Grav. 21, L65 (2004); S. Rasanen, Jour. Cosmol. Astropart. Phys. 0402, 003 (2004); E. W. Kolb, S. Matarrese, A. Notari and A. Riotto, Phys. Rev. D 71, 023524 (2005); K. Nakamura, Prog. Theor. Phys. 113, 481 (2005); P. Martineau and R. Brandenberger, Phys. Rev. D 72, 023507 (2005); K. A. Malik, Jour. Cosmol. Astropart. Phys. 0511, 005 (2005); S. Rasanen, Class. Quant. Grav. 23, 1823 (2006); E. W. Kolb, S. Matarrese and A. Riotto, On cosmic acceleration without dark energy, astro-ph/0506534; P. Martineau and R. Brandenberger, Back-Reaction: A Cosmological Panacea, astro-ph/0510523 K. Nakamura, Second-order Gauge Invariant Cosmological Perturbation Theory: Einstein equations in terms of gauge invariant variables, gr-qc/0605108, T. Buchert, J. Larena and J.-M. Alimi, Correspondence between kinematical backreaction and scalar field cosmologies - the 'morphon field', gr-qc/0606020, these studies have by and large concentrated on either general formal issues or on applications to the growth of fluctuations in cosmology. The results presented in this paper (especially the construction of second order energy-momentum tensors such as that of Eq. (15) via the introduction and then variation of second order gauge invariant actions such as that of Eq. (14), and their use to obtain gauge invariant global integral relations such as that exhibited in Eq. (11) ) are all new to the literature.

[2] S. Weinberg, Gravitation and Cosmology: Principles and Applications of the General Theory of Relativity (Wiley, New York, 1972). 
[3] For an $h_{\mu \nu}$ which obeys the first order $\Delta_{\mu \nu}^{(1)}=-\kappa_{4}^{2} \tau_{\mu \nu}^{(1)}$ in a background which obeys $\Delta_{\mu \nu}^{(0)}=0$, direct evaluation of the second order contribution of $h_{\mu \nu}$ to the full $\Delta_{\mu \nu}^{(2)}$, viz. $\Delta_{\mu \nu}^{(2)}(h)$, is explicitly found to reveal that $\Delta_{\mu \nu}^{(2)}(h)$ is both non-zero and covariantly conserved. It was in trying to reconcile this with the fact that, as such, Eq. (5) itself only leads to the covariant conservation of the full $\Delta_{\mu \nu}^{(2)}$ (and actually even to its vanishing) in solutions to Eq. (4) that engendered the study presented in this paper.

[4] With $\Delta^{(2) \mu \nu}(h)$ already being second order, any change in the coordinate measure or in coordinate derivatives due to the coordinate transformation $x^{\mu} \rightarrow x^{\mu}-\epsilon^{\mu}$ would only affect Eq. (11) in third order.

[5] If a gravitational antenna has a covariantly conserved energy-momentum tensor $\hat{\tau}_{\mu \nu}$, its coupling to the fluctuation $h_{\mu \nu}$ can be described by an action $I=\int d^{4} x(-g)^{1 / 2} h^{\mu \nu} \hat{\tau}_{\mu \nu}$, with this action itself being gauge invariant, since no matter how badly behaved the function $\epsilon_{\mu}$ might be at infinity, once $\hat{\tau}_{\mu \nu}$ is conserved, the integral $\int d^{4} x(-g)^{1 / 2}\left(\nabla^{\mu} \epsilon^{\nu}+\nabla^{\nu} \epsilon^{\mu}\right) \hat{\tau}_{\mu \nu}$ can be reduced to a surface term which will then not contribute to a stationary variation of the action with respect to the fluctuation $h_{\mu \nu}$ in which the surface term is held fixed. For both welland badly-behaved $\epsilon^{\mu}$ the coupling of a gravitational wave to an antenna is thus fully gauge invariant.

[6] D. Bak, D. Cangemi and R. Jackiw, Phys. Rev. D 49, 5173 (1994).

[7] As a check on our calculation, we also obtained this same $t_{\mu \nu}^{(2)}$ by first putting the action of Eq. (14) into the harmonic gauge before doing the variation with respect to $g_{\mu \nu}$.

[8] Transformations of the form $h_{\mu \nu} \rightarrow h_{\mu \nu}+p_{\mu} \epsilon_{\nu}+p_{\nu} \epsilon_{\mu}$ which leave the harmonic gauge condition invariant also leave the harmonic gauge $E^{(2)}$ invariant, just as they should.

[9] The Weinberg prescription and our prescription for $t_{\mu \nu}^{(2)}$ only differ by a two-index object which is not only itself covariantly conserved, but which in addition makes no on-shell $\left(p^{0}=|\bar{p}|\right)$ contribution to $E^{(2)}$ even though the integration in Eq. (18) is only three- rather than fourdimensional.

[10] L. F. Abbott and S. Deser, Nucl. Phys. B 195, 76 (1982).

[11] While having Killing vectors is not mandatory for a general spacetime, all spaces of interest in astrophysics and cosmology do have some.

[12] As with the coordinates, changes to the Killing vector under $x^{\mu} \rightarrow x^{\mu}-\epsilon^{\mu}$ only affect Eq. (20) in third order. 
[13] L. Randall and R. Sundrum, Phys. Rev. Lett. 83, 4690 (1999).

[14] P. D. Mannheim, Brane-Localized Gravity (World Scientific, New Jersey, 2005).

[15] For the $d S_{4}$ brane case the analogous energy integral is cut off at the Cauchy horizon where the relevant $e^{A}$ vanishes.

[16] An analogous discussion of brane-world normalization issues can be found in O. DeWolfe, D. Z. Freedman, S. S. Gubser and A. Karch, Phys. Rev. D 62, 046008 (2000), though with an energy-momentum tensor which differs from the one used here.

[17] Despite being standard, brane-world propagators constructed via sums over normalizable modes actually turn out to not be causal, though closely related ones can be constructed which then are [14].

[18] In passing we note that while we have confined our discussion to gravity theories based on the Einstein equations, the approach of this paper can readily be applied to other metric gravitational theories. For instance, for the conformal gravity theory [a theory which has recently been advanced as an alternative to dark matter and dark energy (P. D. Mannheim, Progress in Particle and Nuclear Physics 56, 340 (2006))], in the tensor $\Delta^{\mu \nu}$ one everywhere replaces $G^{\mu \nu}$ by the equally kinematically covariantly conserved $W^{\mu \nu}=(-g)^{-1 / 2} \delta I_{W} / \delta g_{\mu \nu}$ where $I_{W}=\alpha_{g} \int d^{4} x(-g)^{1 / 2} C_{\mu \nu \sigma \tau} C^{\mu \nu \sigma \tau}$ is the conformal gravity action. 